\title{
DC-DC Buck Converter Driver with Variable Off-Time Peak Current Mode Control
}

Osvaldo Gasparr: ${ }^{1}$, Paolo Del Croce ${ }^{2}$, Andrea Baschirotto ${ }^{1}$

${ }^{1}$ Department of Physics "G.Occhialini", University of Milano, Bicocca, 20126, Italy

${ }^{2}$ IFAT DC ATV BP PD ADL, Infineon Technologies, Villach, 9500, Austria

\begin{tabular}{l} 
A R T I C L E I N F O \\
\hline Article history: \\
Received: 29 August, 2020 \\
Accepted: O1 November, 2020 \\
Online: 20 November, 2020 \\
Keywords: \\
DC-DC Converter \\
Peak Current Mode Control \\
Simulink \\
dSpace
\end{tabular}

\section{Introduction}

Electronic devices in Automotive industries need for power converters to be correctly sourced. Indeed, battery voltage could range between 4.5 to $>27 \mathrm{~V}$, making it unsuitable for driving any load without provoking damages or malfunctions. DC-DC Converters allow to take as an input the car battery voltage and return as an output a controlled voltage or current required by the load. What is more, the DC-DC protects the load against over-current and over-voltage, ensuring its correct operation. Meanwhile, integrated circuits are required to occupy small space and reduce external components to minimize the associated cost. The core of a Buck DC-DC converter is a switch, or Power-DMOS, which is repeatedly turned on and off, allowing the voltage/current to increase/decrease quickly in the neighborhood of a desired value. The circuit that turns on and off the switch is the control circuit [1]. The control could be either made on the output current or voltage. This paper presents a current control loop able to control all the current features (peak, average and ripple) at once. This paper is an extension of work originally presented in the 26th IEEE International Conference on Electronics Circuits and Systems (ICECS) [2].

The solution arises from the well-known Peak-Current-Mode Control (PCMC), [3] operating in Continuous Conduction Mode
(CCM). Instead of having a constant switching period, as it happens in the typical PCMC, the presented control loop has a variable period. In particular, the time in which the power-DMOS stays off, changes accordingly to the calculated current average value, to make it closer to a target desired value. The loop will be now on referred to it as Variable $T_{o f f}$. Moreover, the typical PCMC intrinsically suffers of instability issues directly attributable to the fixed switching frequency [4]. Therefore, by making it variable, such issues are consequently solved. The use of stabilization techniques such as Slope Compensation [4] can be avoided, which increase the complexity of the system without improving the control precision (indeed current features will change accordingly to the input voltage). Also protection against over-current is already guaranteed: indeed the maximum current, namely the peak, is fixed. The proposed system operates with $500 \mathrm{kHz}$ maximum switching frequency, delivering an average output current of $3 \pm 10 \% \mathrm{~A}$ with a load-fixed $4.5 \mathrm{~V}$ output voltage. The current peak will be limited to 3.3A. The application case could be, for instance, a LED driver, where the LED itself, as soon as the correct current is provided, fixes the output voltage. The circuit has been simulated and then tested in laboratory by using an emulator called dSpace. Instead of a LED, in first place has been used as a load a linear component: a $1.5 \Omega$ resistor.

Finally, the current sensing is integrated, with no need for

*Corresponding Author: Osvaldo Gasparri (LC) Italy, o.gasparri@ campus.unimib.it 
an external pad to sense the current, resulting in a lighter circuit. Compared to the most common solutions [5], the here used fullyintegrated Current Sensing technique requires less external components, with consequent lower cost in terms of BoM and board fabrication.

The paper is organized as follows. The algorithm principle is presented in Section II, together with the formula useful for its implementation, described in Section III. The simulation results are shown in Section IV and the conclusions are drawn in Section V.

\section{Variable- $T_{\text {off }}$ PCMC Algorithm}

A Buck DC-DC converter is a device which in principle allows to convert a given input voltage to a lower output voltage by turning on and off a Power-DMOS, able to withstand to high power. In the application at stake from [4.5, 27] $\mathrm{V}$ are obtained $4.5 \mathrm{~V}$ in output by controlling the current, $I_{L}$, flowing into the load. A simple scheme of a Buck converter is given in Figure 1 .

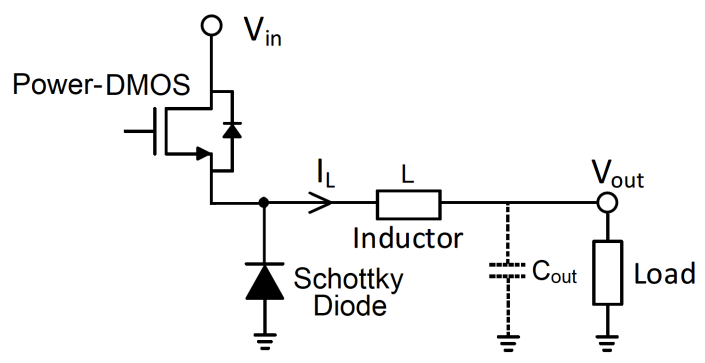

Figure 1: Buck converter scheme

Rather than other diodes, a Schottky diode has been used for its lower threshold $(0.5 \mathrm{~V})$ and higher speed. The presence of the output capacitor, which minimizes the voltage ripple, can be neglected when controlling the current (and the output voltage is fixed by the load) leading to a lighter circuit. The switching period is divided into two phases: the ON-phase when the Power-DMOS conducts $\left(T_{o n}\right)$ and the OFF-phase when the diode conducts $\left(T_{o f f}\right)$. Referring to the Duty-Cycle (D):

$$
\left\{\begin{array}{l}
T_{o n}=D * T \\
T_{o f f}=(1-D) * T \quad \text { with } \quad T=T_{o n}+T_{o f f}
\end{array}\right.
$$

The Buck converter should sink an optimum average current $I_{\text {avg }}$ to make the load work properly and, to avoid damages, the current must fluctuate limitedly. The current ripple should be indeed limited to the average value $\pm 10 \%$, namely $0.3 \mathrm{~A}$. Consequently, the maximum current, the peak value, should be $3.15 \mathrm{~A}$. The rule of the control loop is to ensure that these specifications are met. The relation between the current features can be expressed as follows:

$$
\Delta I_{L}=2 *\left(I_{\max }-I_{\text {avg }}\right)
$$

In standard PCMC, the period $\mathrm{T}$ is fixed. Conversely, in Variable $T_{o f f}$, the period is variable. In particular, $T_{o n}$ changes automatically according to the input voltage, while $T_{o f f}$ is forced to vary every cycle by the control loop, based on the vicinity between the calculated output average value of that cycle and the desired value.
The load current during the ON-phase increases with positive slope given by:

$$
\left(\frac{d I_{L}}{d t}\right)_{O N}=\frac{\Delta I_{L}}{T_{o n}}=\frac{V_{\text {in }}-V_{\text {out }}}{L}
$$

Once $I_{L}$ reaches $I_{\max }$, the power-DMOS turns OFF and the output current decreases with a negative slope:

$$
\left(\frac{d I_{L}}{d t}\right)_{\text {OFF }}=\frac{\Delta I_{L}}{T_{\text {off }}}=-\frac{V_{\text {out }}}{L}
$$

After a time $T_{o f f}$, the DMOS is turned ON again and so on. The output current waveform (equal to the inductor current) has the typical triangular CCM shape as in Figure 2

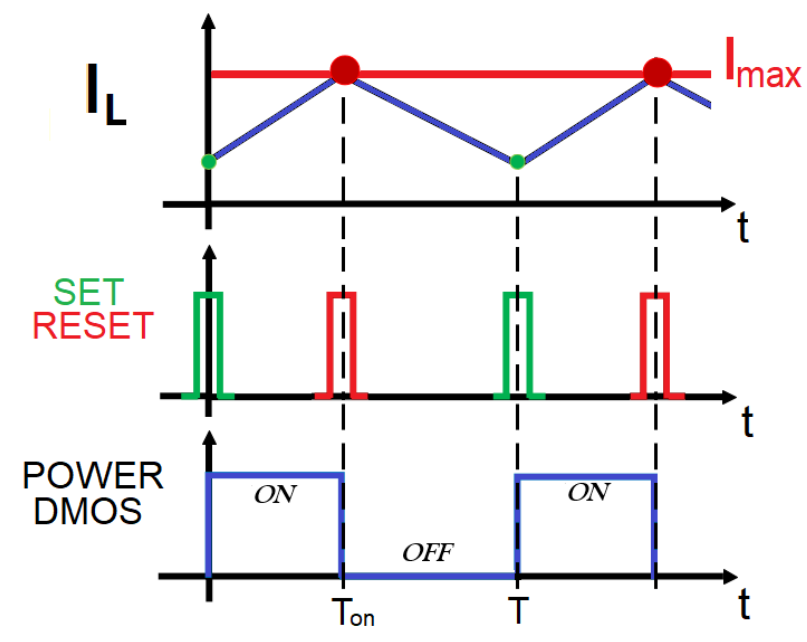

Figure 2: Output current

While $I_{\max }$ is fixed, the $I_{\min }$ is free to change. The loop, by means of a sensing technique, is able to read $I_{\min }$. Indeed, the current sensing happens during the $\mathrm{ON}$ phase to insure the current doesn't grow beyond $I_{\max }$. Therefore, to get $I_{\min }$, it just needed a register to store the first current value when the ON phase begins. Once $I_{\min }$ is known, the loop calculates:

$$
I_{\text {avg }}=\frac{I_{\min }+I_{\max }}{2}
$$

Then, the difference between $I_{\text {avg }}$ and $I_{\text {mean }, \text { ref }}=3 \mathrm{~A}$ gives an error with sign that, once properly amplified by a factor $\mathrm{K}$, is summed to the previous $T_{o f f}$ value. The following $T_{o f f}$ value will be:

$$
T_{\text {off,next }}=T_{\text {off,prev }}+k \cdot\left(I_{\text {avg }}-3 A\right)
$$

In this way, if e.g. $I_{a v g}$ is higher than $3 \mathrm{~A}$, the $T_{\text {off }}$ value will be increased (causing a stretching of the switching period) and allowing the current to decrease more during the OFF phase, reaching a lower $I_{\min }$ with the aim of reducing the average value. If otherwise $I_{\text {avg }}$ is lower than $3 \mathrm{~A}$, the error will be negative, meaning that the $T_{o f f}$ of the next switching cycle must decrease to make the average value higher and matching with the target. The loop operation is resumed in the flow-chart in Figure 3 . The total number of regressions in the algorithm depends on the current rising slope, on the sensing technique sampling frequency and on the counter frequency. Every time a switching cycle ends, the operation restarts from the beginning giving rise to the next switching cycle. 


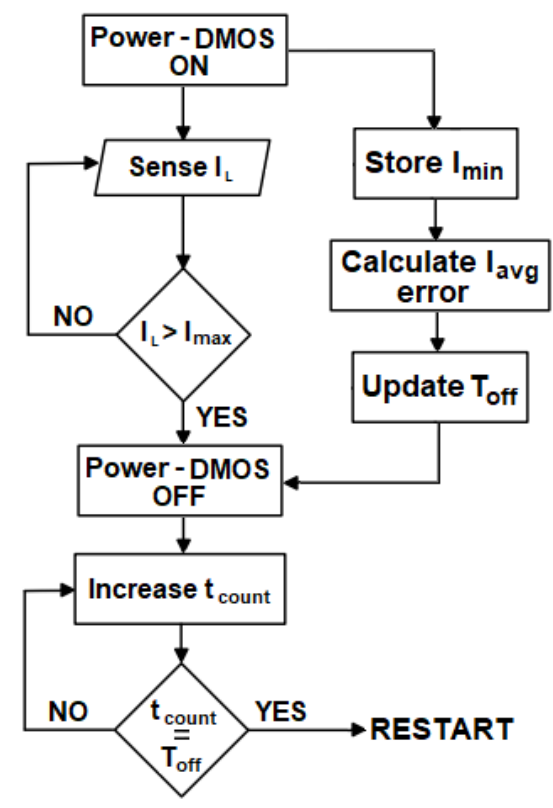

Figure 3: Variable- $T_{\text {off }}$ PCMC algorithm flowchart

While $T_{o f f}$ is decided by the loop, the $T_{\text {on }}$, from $(3)$, changes not only with the the $\Delta I_{L}$-loop dependent- but also with the battery voltage:

$$
T_{\text {on }} \propto \frac{1}{\left(V_{\text {in }}-V_{\text {out }}\right) \cdot \Delta I_{L}}
$$

It should be noticed that a first pre-memorized $T_{\text {off }}$ value guess must exist (to be later overwritten by the cycle) to avoid malfunction at the cranking of the system during the very first switching cycle. This first guess is based on the application: if for instance the desired average is $3 \mathrm{~A}$, the peak is $3.15 \mathrm{~A}$, the inductance $30 \mu \mathrm{H}$ and $V_{\text {out }}=4.5 \mathrm{~V}$, it follows that the $T_{\text {off }}$ value, from (2) and (4), could be grossly chosen as:

$$
T_{o f f}=\frac{L \cdot \Delta I_{L}}{V_{\text {out }}}=4 \mu
$$

Then, given some $V_{\text {out }}$ variations due to the load or some uncertainties related to the inductance value, the references etc., the loop will act as described above, varying the $T_{\text {off }}$ value to better adapt the system to the load specifications.

Moreover, the $T_{\text {off }}$ value has an inferior limit, due to the maximum power-DMOS frequency $\left(f_{\max }\right)$ allowed. Ideally, the $f_{\max }$ can be evaluated for $T_{\text {on }}$ tending to zero, namely:

$$
f_{\text {max }} \simeq \frac{1}{T_{\text {off }}}
$$

In realty, the minimum $T_{\text {on }}$ value won't be zero, but, from (3), for a $V_{i n}=27 \mathrm{~V}$ it will be about $0.8 \mu \mathrm{s}$.

\section{Instability digression}

The typical PCMC loop is just able to control the maximum load current, no matter what ripple and average current are. Therefore, such a solution is not suitable in applications where loads require a precise average current with small shifts around its value. However, the biggest issue of the typical PCMC comes from its instability when the duty cycle grows over $50 \%$ [6]. Every time $T_{o n}>T_{\text {off }}$ or, equivalently, $V_{\text {out }}>V_{\text {in }} / 2$, the system would be unstable. In the application case, where $V_{\text {in }}=[4.5,27] \mathrm{V}$ and $V_{\text {out }}=4.5 \mathrm{~V}$, problems would arise when $V_{\text {in }}<9 \mathrm{~V}$. Figure 4 shows what happen when crossing the limit case $D=0.5$ : if the duty cycle is increased the PCMC is no longer helping in controlling the current. The reason behind this issue is that, in typical PCMC, after a fixed period $T$, the power-DMOS is forced to be turned ON. Instead, in Variable$T_{\text {off }}$, the power-DMOS turns ON $T_{\text {off }}$ seconds after the current has peaked. Contrarily to the typical PCMC, if the input voltage changes, i.e. $T_{\text {on }}$ changes, the $T_{\text {off }}$ doesn't change (4), the ripple doesn't change and therefore the current features are not impacted. The system stays stable. Techniques as Slope compensation [4] are no longer needed.

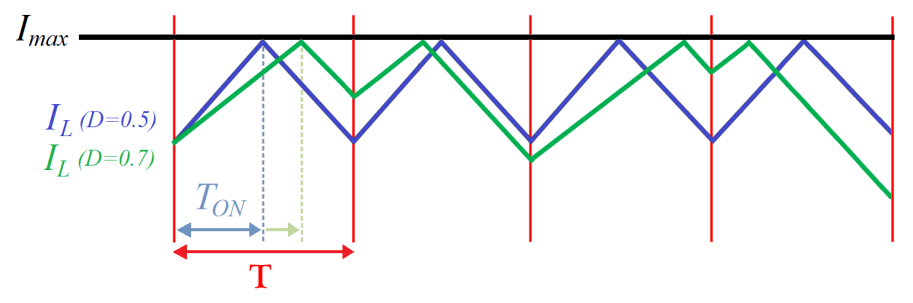

Figure 4: Output current in typical PCMC

\section{Constant- $T_{\text {off }}$ Algorithm Implementa- tion}

The circuit implementation is shown in Figure 5 The only external components are the inductor (L) and the Schottky Diode. As internal components, enclosed in blocks, there are: the Current Sensing, the Control Logic blocks, the Protection, the Internal Reference (including a bandgap voltage reference to generate $I_{\text {max }}$ and $I_{\text {mean }}$ ), the Gate Driver to turn ON and OFF the Power-DMOS [7] and the Power Supply to generate a low voltage domain for the low-side circuit.

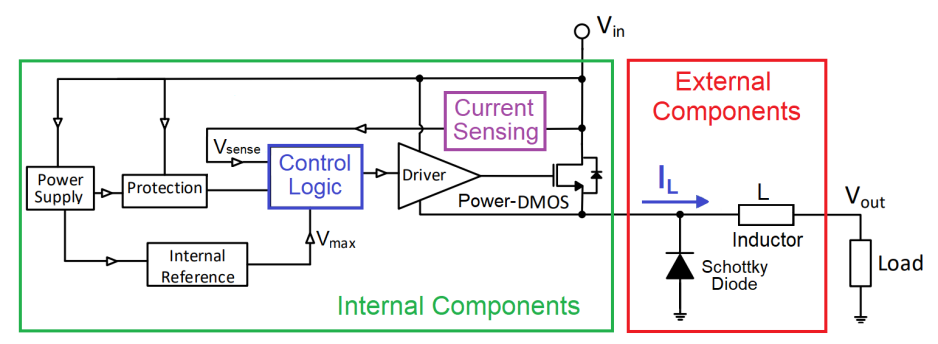

Figure 5: Concept top level

The Current Sensing block is used to read the current flowing $I_{L}$. The Power-DMOS current is here mirrored with a factor $N=1 / 1000$ and flows through a sensing resistance. Based on the read voltage drop, the system is able to determine the magnitude of the current. Since the proposed technique only operates during the ON-phase (when the Power-DMOS conducts) the power consumption is lower than in the case on which the current is sensed on 
the output node, always operating. Furthermore, an internal sensing block also allows to minimize the area occupation on silicon.

\subsection{Control Logic block}

Figure 6 shows the concept Control Logic block implementation. The result of the comparison between $I_{\text {sense }}$ and $I_{\max }$ gives the Reset of the SR Latch. When $I_{\text {sense }} \geq I_{\max }$, the Reset activates and the Power-DMOS turns OFF through the Gate Driver. The Set input is instead connected to a counter consisting of an oscillator and a chain of four D Flip-Flops. As soon as the Power-DMOS turns OFF, the counter starts counting up to $T_{\text {off }}$ duration. Afterwards the Set is activated and the Power-DMOS turns ON. The current value at this moment, $I_{\min }$, is stored into a register. The loop then calculates the average error on respect to the desired $I_{\text {mean }}$ value. The error, summed to the previous $T_{\text {off,prev }}$ value returns the $T_{\text {off,next }}$ as in (6).

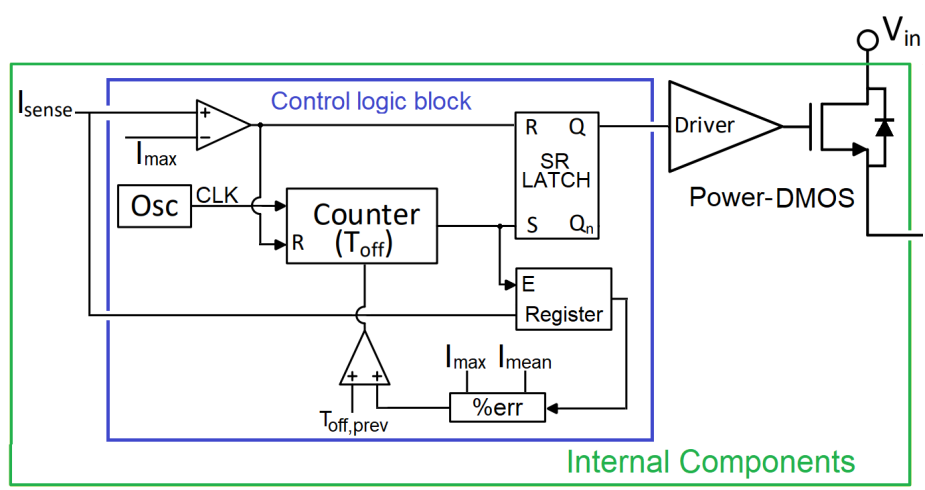

Figure 6: Variable $T_{\text {off }}$ Current Control Loop implementation

\section{Design example and Simulations results}

Table 1 resumes the application specs.

Table 1: Summary of specifications

\begin{tabular}{|c|c|}
\hline \hline Technology & $0.35 \mu \mathrm{m}$ \\
\hline $\mathbf{V}_{\text {in }}$ & $4.5-27 \mathrm{~V}$ \\
\hline $\mathbf{V}_{\text {out }}$ & $4.5 \mathrm{~V}$ \\
\hline $\mathbf{f}_{\text {max }}$ & $500 \mathrm{kHz}$ \\
\hline Maximum Current Level & $3.3 \mathrm{~A}$ \\
\hline Mean Current Level & $3 \mathrm{~A}$ \\
\hline Output Current Ripple & $0.60 \mathrm{~A}$ \\
\hline
\end{tabular}

By applying the formulas given in Section I, the design parameters are obtained. (9) gives the lower $T_{\text {off }}$ limit:

$$
T_{\text {off }} \simeq \frac{1}{500 \mathrm{kHz}}=2 \mu \mathrm{s}
$$

In fact, $1 / f_{\max }=T_{o n, \min }+T_{o f f}$ and $(3)$ becomes:

$$
V_{\text {out }} \cdot T_{\text {off }}=\left(V_{\text {in, } \max }-V_{\text {out }}\right) \cdot\left(1 / f_{s w, \max }-T_{\text {off }}\right)
$$

Therefore a more precise estimation of the minimum $T_{\text {off }}$ value is given by:

$$
T_{o f f}=\frac{27 \mathrm{~V}-4.5 \mathrm{~V}}{27 \mathrm{~V}} \cdot \frac{1}{500 \mathrm{kHz}}=1.7 \mu \mathrm{s}
$$

Once the ripple (Table 1) and $T_{\text {off }}(12)$ are given, both the peak and the average current are fixed. Furthermore, by using these parameters in (4), the inductance value can be chosen as:

$$
L>\frac{V_{\text {out }} * T_{\text {off }}}{\Delta I_{L}}=13 \mu H \rightarrow 30 \mu H
$$

(to stay large, considering also $20 \%$ of inductance value uncertainty on the nominal value and considering that actually the typical operation is centered around $V_{i n}=16 \mathrm{~V}$ ).

\subsection{Simulation results}

The concept of Figure 5 has been firstly implemented on Simulink (MATLAB) to ideally simulate the behaviour of the high-level concept design. Simulink works with a block approach system, shown in Figure 7 which follows exactly from Figure 5 . Other software are actually exploited in the design phase (such as Virtuoso-cadence). However, there, the circuit must be designed in a deeper transistorlevel. Since the design may take large amounts of time, prototyping must be done previously ensuring that the chosen topology is the most suitable one for the application in question and that its worthwhile to proceed with transistor-level design and future fabrication.
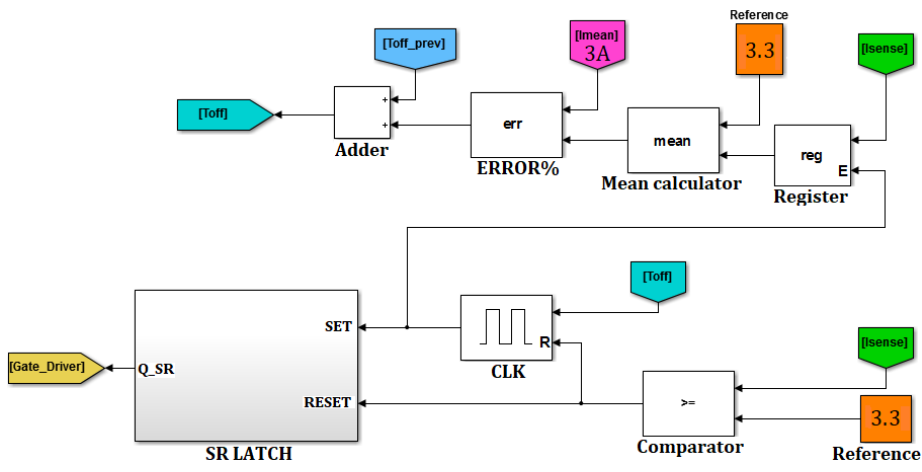

Figure 7: Simulink implementation

The simulations include also worst case scenarios to ensure the correct operation in each condition. To simulate the presence of a load which fixes the $4.5 \mathrm{~V}$ output voltage with a $3 \mathrm{~A}$ average current, a $1.5 \Omega$ resistor has been used.

Figure 8 (left) shows how the system works with a 7V input voltage and a peak reference reduced from $3.3 \mathrm{~A}$ to $3.2 \mathrm{~A}$, i.e. with a $\Delta_{I_{L}}=0.4 \mathrm{~A}$. As expected, the operation is not affected by instability issues which, in a typical PCMC, would surely arise whenever $V_{i n}<9 \mathrm{~V}$. Later, the input voltage has been abruptly increased to $18 \mathrm{~V}$ to also prove that a $V_{\text {in }}$ change (as could happen during the cranking of the car) has no relevant effect on the operation. The $T_{o f f}$ remains unchanged (no dependency on the $V_{i n}$ ) and equal to $2.7 \mu \mathrm{s}$ as expected from (7) (with $\Delta_{I_{L}}=0.4 \mathrm{~A}$ ). 


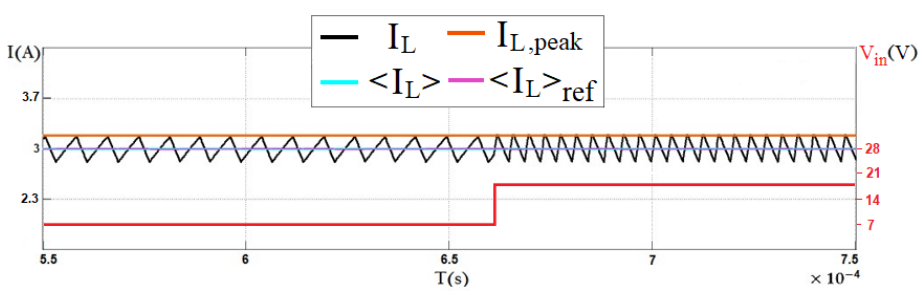

Figure 8: System response changing the input voltage

Figure 9 shows instead the transient simulation plot for $V_{i n}=$ $18 \mathrm{~V}$ with the peak reference at $3.3 \mathrm{~A}$ as from specification. As expected from (7), the $T_{o f f}=4 \mu \mathrm{s}$. To further prove the system robustness, an average reference positive step (from $3 \mathrm{~A}$ to $3.15 \mathrm{~A}$ ) has been added. In order to achieve the new average current, the loop gradually reduces the $T_{\text {off }}$ to make the calculated average equal to the target. Since the peak reference stays equal, according to 8 and (9), the ripple reduces and the switching frequency increases up to $260 \mathrm{kHz}$.

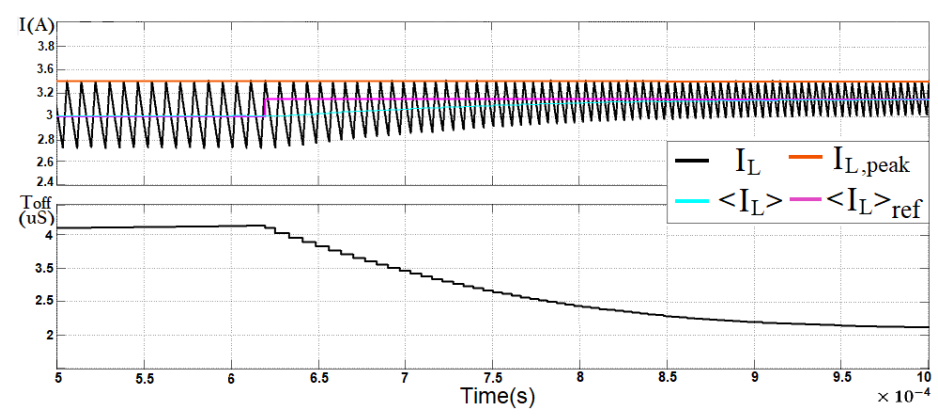

Figure 9: System response changing the average reference

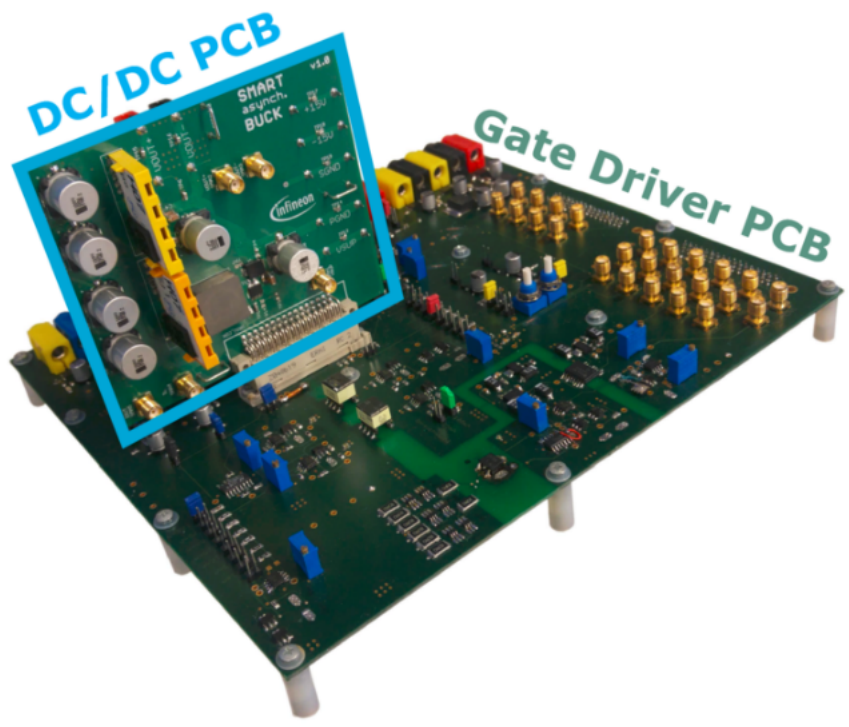

Figure 10: Buck Converter and Gate Driver PCBs

Once proven the concept works as expected, the system can be tested in laboratory by means of a rapid control prototyping called dSpace. Here, the power-DMOS and external components are no longer digital, but located on a DC-DC converter Printed Circuit
Board (PCB), which is connected to a Gate driver board (Figure 10, connected in turn to dSpace.

dSpace, through its FPGA, will embody the Simulink bloch approach control logic circuit (uploaded by PC), also providing the current sensing, the internal reference and all the devices to communicate with the hardware (ADCs, DACs). dSpace allows to test whatever control circuit including all the un-idealities of the system, verifying moreover the correct operation of the power-DMOS and that one of a real load. In this way it can be eventully proven that, not only the circuit works as expected, but that is suitable for the application at stake. Through a Graphic User Interface, in Figure 11 the user can monitor the system operation using the PC. Not only to insert the protection to avoid $\mathrm{OC}$ and $\mathrm{OV}$, but also to vary the references and other parameters and observe the system response and waveforms in each condition.

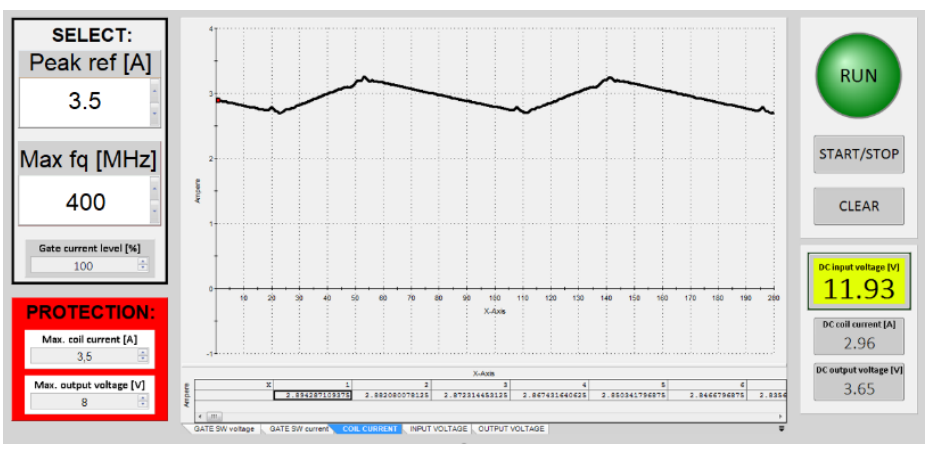

Figure 11: Graphic User Interface

Of course, for having a higher sampling precision, the same waveform can be actually taken from oscilloscope, shown in Figure 12. As expected, the circuit is able to drive the load maintain the desired current features over time and with the same theoretical behaviour of Figure 2

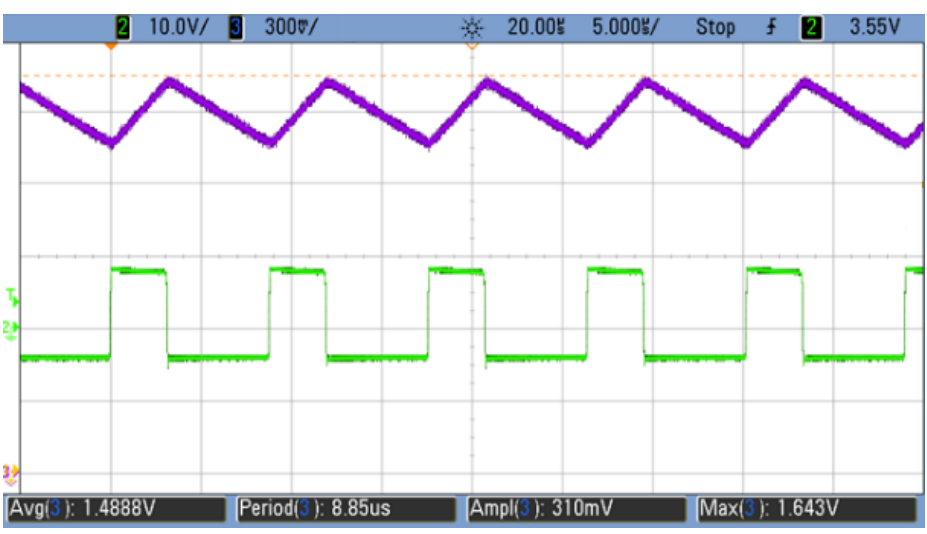

Figure 12: Output current (violet) and gate driver (green) Waveforms from oscilloscope

\section{Conclusions}

The presented DC-DC Buck Converter Variable- $T_{\text {off }}$ loop allows to precisely and smartly control the current features, returning a reliable car battery sourced driver. Other than the current peak, the 
loop is able to control the average current. Moreover, given (2), also the ripple is indirectly controlled. With respect to other solutions, the switching period is variable, intrinsically solving the instability issues. Indeed, the $T_{\text {on }}$ changes with the input voltage while $T_{\text {off }}$ increases/decreases at each switching cycle accordingly to the comparison between $I_{\text {avg }}$ and the target value, making (2) smaller/bigger (i.e. increasing/decreasing $I_{\text {avg }}$ ).

The system exploits an integrated low-side sensing resistor circuit to minimize the Printed Circuit Board usage space, avoiding extra pads to sense the current. To make the chip lighter and cheaper, the Buck Converter $C_{\text {out }}$ has been neglected, as superfluous for the application.

The concept has been simulated with Simulink and then a more rigorous test has been conducted in laboratory with dSpace where worst condition simulations shown an excellent dynamic behaviour. The circuit appears to be robust, proving that sudden perturbations on $V_{\text {in }}, I_{\max }$ and $T_{\text {off }}$ do not upset the operation of the circuit. Compared to [8], the presented solution is lighter and works with an input voltage range of $[4.5,27] \mathrm{V}$, able to drive a $3 \mathrm{~A}$ LED with $500 \mathrm{kHz}$ maximum switching frequency, allowing extremely good average regulation precision and speed. A more detailed comparison of all Buck performances, including efficiency, with the state of the art is provided in [7].

Acknowledgment A part of the work has been performed in the project iDev40. The iDev40 project has received funding from the ECSEL Joint Undertaking (JU) under grant agreement No 783163. The JU receives support from the European Union's Horizon 2020 research and innovation programme. It is co-funded by the consortium members, grants from Austria, Germany, Belgium, Italy, Spain and Romania.The information and results set out in this publication are those of the authors and do not necessarily reflect the opinion of the ECSEL Joint Undertaking.

\section{References}

[1] L. Dixon, "Average Current Mode Control of Switching Power Supplies," 1999.

[2] O. Gasparri, R. Di Lorenzo, P. Del Croce, A. Pidutti, A. Baschirotto, "Variable off-Time Peak Current Mode Control (VoT-PCMC) as method for average current regulation in Buck Converter Drivers," 2019.

[3] I. J. Prasuna, M. Kavya, K. Suryanarayana, B. Shrinivasa Rao, "Digital Peak Current Mode Control of Boost Converter," 2014.

[4] Z. Jiuming, L. Shulin, "Design of slope compensation circuit in peak-current controlled mode converters," 1310-1313, 2011.

[5] J. Ejury, Buck Converter Design, Infineon Technologies North America (IFNA), 2013.

[6] Fang, Chung-Chieh, Redl, "Subharmonic Instability Limits for the Peak-CurrentControlled Buck converter With Closed Voltage Feedback Loop," Power Electronics, 1085-1092, 2015.

[7] R. Di Lorenzo, O. Gasparri, A. Pidutti, P. Del Croce, A. Baschirotto, "On-Chip Power Stage and Gate Driver for Fast Switching Applications," 2019.

[8] V. Anghel, C. Bartholomeusz, A. Vasilica, G. Pristavu, G. Brezeanu, "Variable Off-Time Control Loop for Current-Mode Floating Buck Converters in LED Driving Applications," 2014. 